\title{
Prognosis and risk factors for the development of pulmonary metastases after preoperative chemoradiotherapy and radical resection in patients with locally advanced rectal cancer
}

\author{
Weihao Li", Jianhong Peng", Cong Li, Lifang Yuan, Wenhua Fan, Zhizhong Pan, Xiaojun Wu, Junzhong Lin \\ Department of Colorectal Surgery, Sun Yat-sen University Cancer Center, State Key Laboratory of Oncology in South China, Collaborative \\ Innovation Center for Cancer Medicine, Guangzhou 510060, China \\ Contributions: (I) Conception and design: W Li, J Peng, X Wu, J Lin; (II) Administrative support: All authors; (III) Provision of study materials or \\ patients: C Li, L Yuan, W Fan, Z Pan, X Wu, J Lin; (IV) Collection and assembly of data: J Peng, W Li; (V) Data analysis and interpretation: W Li, \\ J Peng; (VI) Manuscript writing: All authors; (VII) Final approval of manuscript: All authors. \\ \#These authors contributed equally to this work. \\ Correspondence to: Junzhong Lin; Xiaojun Wu. Department of Colorectal Surgery, Cancer Center, Sun Yat-sen University, 651 Dongfeng Road East, \\ Guangzhou 510060, China. Email: linjzh@sysucc.org.cn; wuxj@sysucc.org.cn.
}

Background: Although preoperative chemoradiotherapy (CRT) followed by total mesorectal excision (TME) is currently considered effective for treating locally advanced rectal cancer (LARC), a proportion of patients develop postoperative pulmonary metastases. The current study aimed to assess the prognostic characteristics and risk factors for the development of rectal cancer pulmonary metastases after CRT and radical resection.

Methods: We retrospectively analyzed data collected on 544 consecutive patients who were diagnosed with LARC and underwent preoperative CRT followed by tumor radical resection between December 2003 and June 2014. Overall survival (OS), disease-free survival (DFS), and pulmonary metastasis rates were calculated and compared among the subgroups, and risk factors for pulmonary metastases were identified by Cox models.

Results: A total of $61(11.2 \%)$ patients developed pulmonary metastases postoperatively, 45 of whom (73.8\%) developed the condition in the first 24 months. The 1-, 2-, and 3-year pulmonary metastasis rates were $6.7 \%, 10.4 \%$, and $11.7 \%$, respectively. Compared with the disease-free group, the pulmonary metastases group had a significantly lower proportion of downstaging and pathological complete regression (pCR) rate and a significantly higher proportion of low rectum tumor. In multivariate analysis, a distance of the tumor $\leq 5 \mathrm{~cm}$ from the anal verge [hazard ratio (HR), 1.394; 95\% confidence interval (CI), 1.211-3.736; $\mathrm{P}=0.003]$ was identified as an independent negative predictor of the 3 -year pulmonary metastasis rate, and N0 stage (HR, 0.490; 95\% CI, 0.261-0.919; P=0.026) and TNM downstaging (HR, 0.514; 95\% CI, 0.2650.997; $\mathrm{P}=0.049$ ) were identified as independent positive predictors of the 3 -year pulmonary metastasis rate.

Conclusions: Pulmonary metastases warranted a more intensive follow-up in patients with low rectal cancer, lymph node metastases and poor response after preoperative CRT and radical tumor resection.

Keywords: Local advanced rectal cancer; pulmonary metastasis; preoperative chemoradiotherapy; risk factors; prognosis

Submitted Jun 24, 2019. Accepted for publication Nov 15, 2019.

doi: 10.21037/atm.2019.12.108

View this article at: http://dx.doi.org/10.21037/atm.2019.12.108 


\section{Introduction}

Colorectal cancer (CRC) is the third most common cancer and one of the leading causes of cancer death and seriously decreases life expectancy worldwide and in China $(1,2)$. The lungs are the most common extra-abdominal organ in which CRC metastasizes (3). In recent years, with the development of the increasing accuracy and widespread use of chest computed tomography (CT) at the initial diagnosis and follow-up, the proportion of CRC patients diagnosed with pulmonary metastasis is increasing, and is approximately $24.5 \%$ to $29.2 \%$ (1,4-10). Moreover, previous studies have reported that patients with rectal cancer are more likely to develop pulmonary metastasis than those with colon cancer $(3,10)$. Therefore, the diagnosis and treatment of rectal cancer pulmonary metastases is an extremely important clinical problem.

Preoperative chemoradiotherapy (CRT) followed by total mesorectal excision (TME) is currently considered the standard treatment for locally advanced rectal cancer (LARC) (11-15). Oncological outcomes have been found to be satisfactory with this strategy, achieving a $66 \%$ to $75 \% 5$-year overall survival (OS) rate with a low local recurrence rate between $6 \%$ and $8 \%(11,12,16)$. Moreover, $15 \%$ to $20 \%$ of affected patients were able to obtain a pathological complete response (pCR) (17-19). Nevertheless, we previously reported that approximately $20 \%$ of patients failed to benefit from preoperative CRT and developed postoperative distant metastasis, in which over $50 \%$ of metastatic organs were the lungs (20). Compared with liver or peritoneal metastasis, pulmonary metastasis has a relatively slower growth rate and a better overall prognosis (21), indicating different metastasis patterns warrant different treatment strategies. However, few studies have evaluated the characteristics of pulmonary metastasis after CRT followed by TME in LARC patients, and this issue therefore needs further description and study.

In this study, we aimed to assess the clinical and prognostic characteristics and risk factors for the development of rectal cancer pulmonary metastases after preoperative CRT and TME to determine the clinical implications of rectal pulmonary metastases among patients who undergo curative treatment.

\section{Methods}

\section{Patient selection}

This study included a total of 577 consecutive patients with rectal cancer who underwent preoperative CRT followed by TME from December 2003 through June 2014 at Sun Yat-sen University Cancer Center, China. The following inclusion criteria were used: (I) histologically confirmed rectal adenocarcinoma; (II) cT3-4 or N+ disease before CRT; (III) no other anti-tumor therapy was received before CRT; and (IV) no distant metastatic disease before TME. Thirty-one patients were excluded from this study due to distant metastatic disease before CRT, and an additional two patients who were confirmed to have adenosquamous carcinoma were also excluded. This left a total of 544 individuals who were included in the analysis. The patient demographics, tumor characteristics, neoadjuvant chemotherapy cycles, radiotherapy doses, surgery records and follow-up results were carefully reviewed. The present study was performed according to the ethical standards of the World Medical Association Declaration of Helsinki and approved by the Institutional Review Board and Independent Ethics Committees of Sun Yat-sen University Cancer Center. The informed consent requirement was waived based on the nature of this retrospective study, in which patient data were kept confidential.

\section{Treatment}

Neoadjuvant chemotherapy with XELOX (oxaliplatin $130 \mathrm{mg} / \mathrm{m}^{2}$ administered intravenously on day 1 and capecitabine $1000 \mathrm{mg} / \mathrm{m}^{2}$ administered orally twice daily on days 1-14 for a 3-week cycle) or a modified FOLFOX6 (oxaliplatin $85 \mathrm{mg} / \mathrm{m}^{2}$ in a $2-\mathrm{h}$ infusion, bolus fluorouracil $400 \mathrm{mg} / \mathrm{m}^{2}$ on day 1 , and a $46-\mathrm{h}$ infusion of fluorouracil $2,400 \mathrm{mg} / \mathrm{m}^{2}$ ) regimen. All patients received concurrent radiotherapy of 50 Gy delivered over 5 weeks in fractions of 2.0 Gy daily on 5 consecutive days per week. All patients underwent resection of the primary tumor with lymph node dissection based on the TME technique 6-8 weeks after the completion of preoperative radiotherapy. Lateral pelvic lymph node dissection was not routinely performed except in cases of valid imaging evidence. Adjuvant chemotherapy, including XELOX and modified FOLFOX6 regimens, was scheduled to begin within 3-6 weeks postoperatively. The treatment strategy and operability of pulmonary metastases for each patient were determined according to the final agreement of the multidisciplinary team (MDT).

\section{Definition and measurements}

Pathological assessments and staging of the resected specimens were confirmed according to tumor-node- 
metastasis (TNM) classification by two independent pathologists. pCR was defined as the absence of viable tumor cells, with only fibrotic masses or acellular mucin pools present in proximity to the primary tumor and lymph nodes. Pulmonary metastasis diagnosis was mainly dependent on radiological evidence suggestive of pulmonary metastasis. Findings on CT were agreed upon by at least two independent radiologists. Pulmonary metastases group included the patients with only pulmonary metastases or concurrent extrathoracic metastases at the first disease progression after surgery. Other recurrence group included patients with other extrathoracic metastases or local recurrence without pulmonary metastases at the first disease progression after surgery. The response was defined according to response evaluation criteria in solid tumors (RECIST) standard 1.1.

\section{Follow-up}

Overall survival (OS) was defined as the interval from the date of surgery until death of any cause or the last follow-up; patients without any event (metastasis or death) at the last follow-up date were censored. Disease-free survival (DFS) was defined as the interval from surgery to disease recurrence, death, or the last follow-up. The pulmonary metastasis rate was defined as the cumulative incidence of pulmonary metastasis. All patients were observed through subsequent visits every 3 months for 2 years and then semiannually until 3 years after surgery. Physical examination, blood tests for carcinoembryonic antigen (CEA) and carbohydrate antigen 19-9 (CA19-9) levels, abdominal ultrasonography, and chest X-rays were conducted every 3 months postoperatively. Chest/ abdominal/pelvic computed tomography (CT) and colonoscopy were performed annually. The last follow-up visit was performed in December 2018.

\section{Statistical analysis}

All statistical analyses were performed using IBM SPSS statistics software, version 21.0 (IBM Corp., Armonk, NY, USA). Categorical variables were given as percentages and compared using the Chi-square or Fisher's exact test when appropriate. The OS, DFS and pulmonary metastasis rates were estimated with the Kaplan-Meier method, and the differences between groups were then assessed with the log-rank test. Parameters for which $\mathrm{P}<0.05$ for the 3 -year pulmonary metastasis rate in the univariate Cox models were further assessed in multivariate Cox models using a forward stepwise method. Hazard ratios (HRs) and 95\% confidence intervals (CIs) were subsequently calculated. In addition, patients in other recurrence groups were excluded from the univariate and multivariate Cox models. All of the statistical tests were two-sided, and $\mathrm{P}<0.05$ was considered significant.

\section{Results}

\section{Characteristics of the whole study population}

Among the total of 544 patients, 361 (66.4\%) were males, and $183(33.6 \%)$ were females, and they had a median age of 55 years old (range, 19-85 years old). With respect to postoperative pathological staging, 377 (69.3\%) patients were identified as downstaging, of which 132 (24.3\%) patients were identified as pCR. There were 442 patients who received adjuvant chemotherapy after surgery resection. The patients were followed for a median of 36.9 months (range, 8.0-130.0 months). Overall, 96 (17.6\%) patients died from the disease, 27 (5.2\%) were alive with recurrence, and $421(77.4 \%)$ were disease-free at the end of followup. In total, 123 (22.6\%) patients developed postoperative metastases or local recurrence during the study period, including $61(52.0 \%)$ with pulmonary metastases, 31 (25.2\%) with liver metastases, 11 (8.9\%) with extensive dissemination of the abdominal cavity, 8 (6.5\%) with bone metastases, $7(5.7 \%)$ with brain metastases and $5(4.1 \%)$ with local recurrence.

\section{Survival analysis}

As shown in Figure 1A, the 3-year OS and DFS rates were $91.5 \%$ and $89.3 \%$, respectively, in the entire study population. As shown in Figure 1B, the 3-year OS rates were significantly lower in the pulmonary metastases group than in the disease-free group but similar between the pulmonary metastases group and other recurrence group $(53.7 \%$ vs. 97.1\%; $\mathrm{P}<0.001 ; 53.7 \%$ vs. $44.9 \% ; \mathrm{P}=0.93)$.

\section{Characteristics of patients who developed pulmonary metastases}

The median time to development of pulmonary metastases from surgery was 13.0 months (range, 3.0-52.0 months). Forty-five $(80.3 \%)$ patients developed pulmonary only metastasis, while 12 (19.7\%) patients developed concurrent 

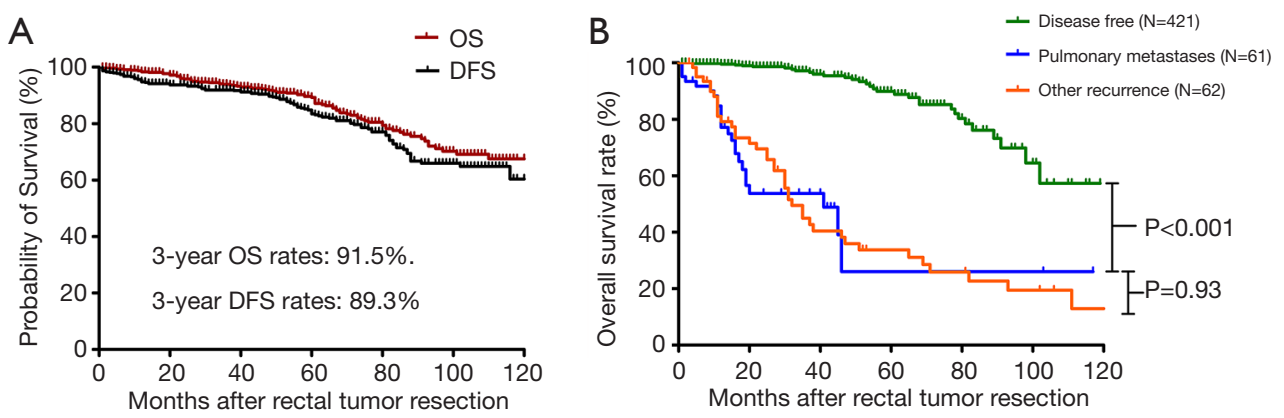

Figure 1 Kaplan-Meier curves of the patients with locally advanced rectal cancer (LARC) receiving preoperative chemoradiotherapy. (A) The whole study population; (B) comparison of overall survival (OS) among the disease free group, pulmonary metastases group and the other recurrence group.
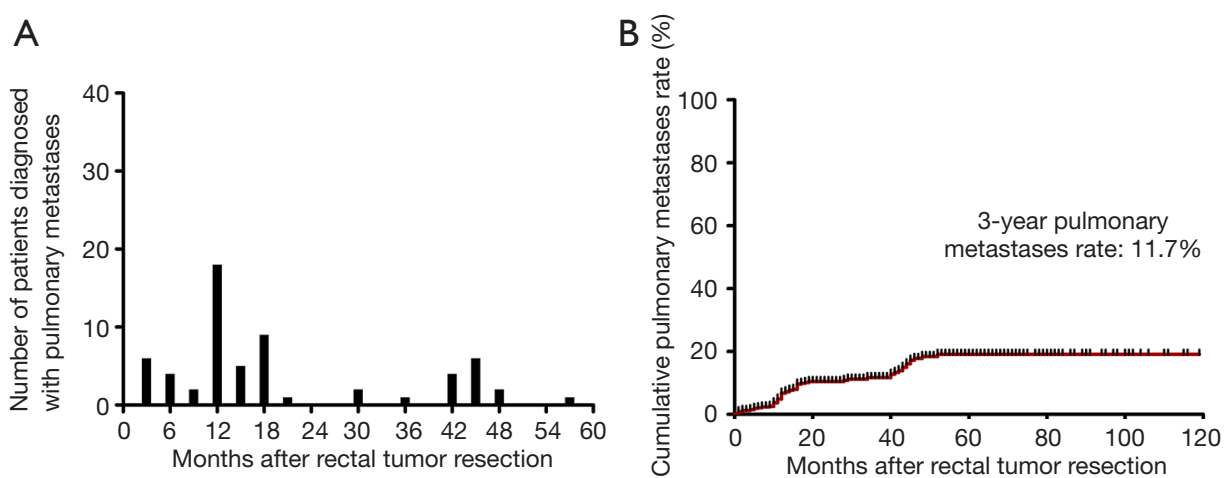

Figure 2 Description of the development of pulmonary metastases in the patients over time after rectal tumor resection. (A) The number of the patients diagnosed with pulmonary metastases after rectal tumor resection over time; (B) cumulative pulmonary metastases rate of the patients diagnosed with pulmonary metastases over time (patients developing other postoperative recurrence were excluded).

extrathoracic metastases or local recurrence, including 8 patients with liver metastases, 2 with brain metastases, and 2 with local recurrence. Among them, 25 patients (41.0\%) presented unilateral pulmonary metastasis, while 36 patients (59.0\%) had bilateral pulmonary metastases. Forty-two patients $(68.9 \%)$ received chemotherapy as the treatment for pulmonary metastases and 11 patients $(18.0 \%)$ received surgery or ablation. The association between number of patients diagnosed with pulmonary metastases and the time after surgery is shown in Figure $2 A$; we found that the highest peak was during 12 to 18 months and the second peak was during 42 to 48 months. Forty-five patients $(73.8 \%)$ developed pulmonary metastases in the first 24 months. In Figure 2B, the 1-, 2-, 3-, and 5-year pulmonary metastasis rates were $6.7 \%, 10.4 \%, 11.7 \%$, and $19.1 \%$, respectively.

\section{Relationships between the development of rectal cancer pulmonary metastases and clinicopathological characteristics}

The clinicopathological and treatment information of all patients are summarized in Tables 1,2. The proportion of patients with tumors $\leq 5 \mathrm{~cm}$ from the anal verge was higher in patients in the pulmonary metastases group than in the disease-free group (78.7\% vs. $52.3 \% ; \mathrm{P}=0.001)$ or the other recurrence group (78.7\% vs. $59.7 \% ; \mathrm{P}=0.045)$. Patients in the pulmonary metastases group were less likely to have completed neoadjuvant chemotherapy (receiving only $1-2$ cycles) than were those in the disease-free group (72.1\% vs. $53.9 \% ; \mathrm{P}=0.026)$. TNM downstaging rate, response rate and $\mathrm{pCR}$ rate were lower in patients in the pulmonary metastases group than in those in the disease- 
Table 1 Comparison of characteristics of the patients with disease free or with pulmonary metastases

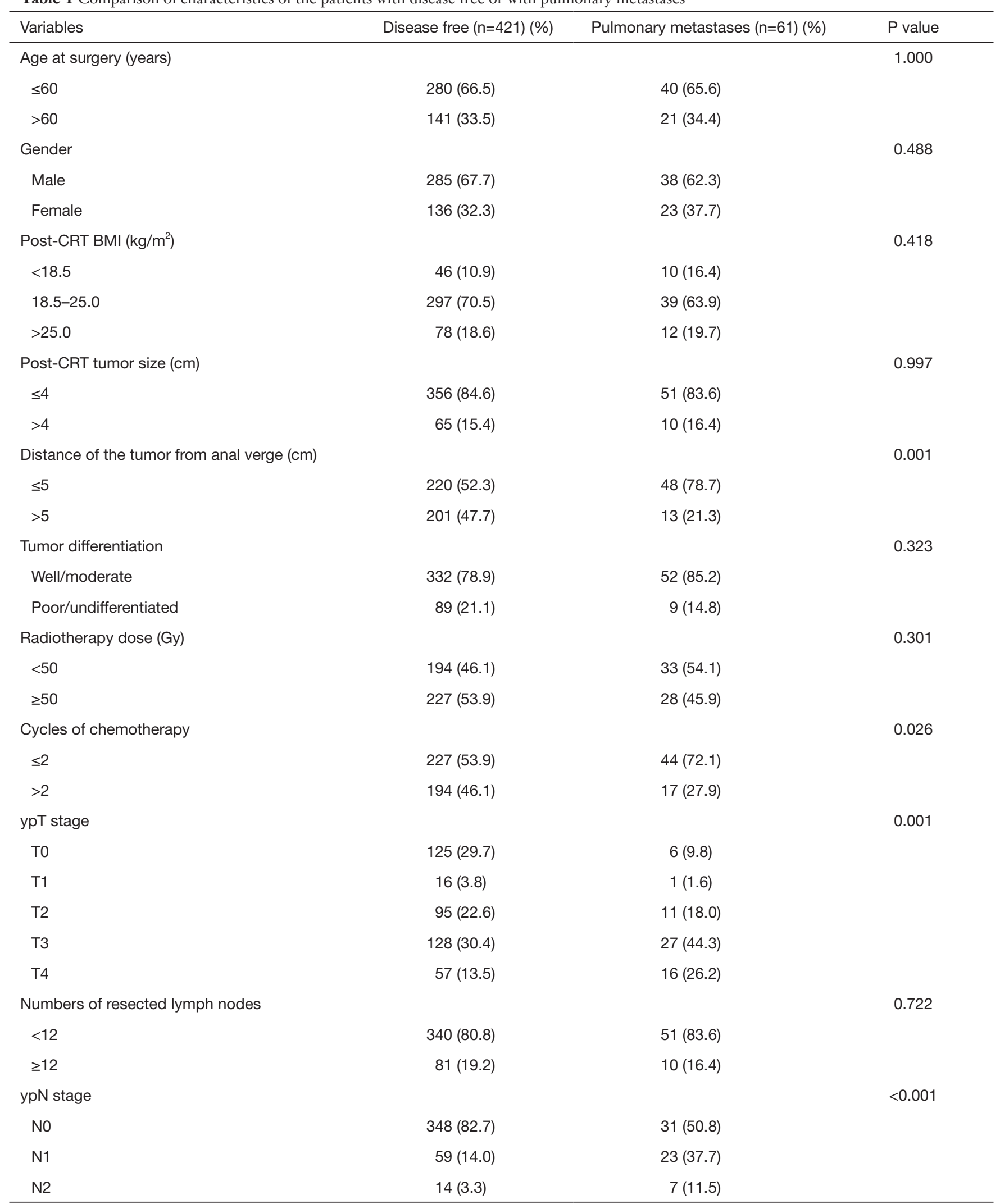

Table 1 (Continued) 
Table 1 (Continued)

\begin{tabular}{|c|c|c|c|}
\hline Variables & Disease free $(n=421)(\%)$ & Pulmonary metastases $(n=61)(\%)$ & $P$ value \\
\hline ypTNM stage & & & $<0.001$ \\
\hline 0 & $120(28.5)$ & $6(9.8)$ & \\
\hline I & $97(23.0)$ & $5(8.2)$ & \\
\hline ॥ & $131(31.1)$ & $20(32.8)$ & \\
\hline Yes & $322(76.5)$ & $24(39.3)$ & \\
\hline No & $99(23.5)$ & $37(60.7)$ & \\
\hline Treatment evaluation & & & 0.037 \\
\hline $\mathrm{CR}$ & $19(4.5)$ & $3(4.9)$ & \\
\hline PD & $3(0.7)$ & $1(1.6)$ & \\
\hline \multicolumn{2}{|c|}{ Interval between completion of CRT and surgery (weeks) } & & 1.000 \\
\hline$\leq 8$ & $281(66.7)$ & $41(67.2)$ & \\
\hline$>8$ & $140(33.3)$ & $20(32.8)$ & \\
\hline Surgical procedure & & & 0.784 \\
\hline LAR or CAA & $257(61.0)$ & $35(57.4)$ & \\
\hline APR & $144(34.2)$ & $22(36.1)$ & \\
\hline Hartmann & $20(4.8)$ & $4(6.6)$ & \\
\hline$\leq 5$ & $400(95.0)$ & $48(78.7)$ & \\
\hline$>5$ & $21(5.0)$ & $13(21.3)$ & \\
\hline Preoperative (post-CRT) serum CA19-9 (U/mL) & & & 0.519 \\
\hline$\leq 35$ & $195(46.3)$ & $25(41.0)$ & \\
\hline$>35$ & $226(53.7)$ & $36(59.0)$ & \\
\hline Adjuvant chemotherapy & & & 0.969 \\
\hline Yes & $343(81.5)$ & $49(80.3)$ & \\
\hline No & $78(18.5)$ & $12(19.7)$ & \\
\hline
\end{tabular}

\#, there were totally 53 patients (9.7\%) in disease free group and 15 patients (2.8\%) in pulmonary metastases group developing surgical complications; *, other surgical complications include anastomotic bleeding, obstruction, perforation, sexual dysfunction, defecation disorder, Uropoiesis dysfunction. CRT, chemoradiotherapy; BMI, body mass index; CR, complete response; PR, partial response; SD, stable disease; PD, progressive disease; LAR, low anterior resection; CAA, Coloanal Anastomosis; APR, Abdominoperineal Resection; CEA, carcinoembryonic antigen; CA19-9, carbohydrate antigen 19-9. 
Table 2 Comparison of characteristics of the patients grouped by different metastatic pattern

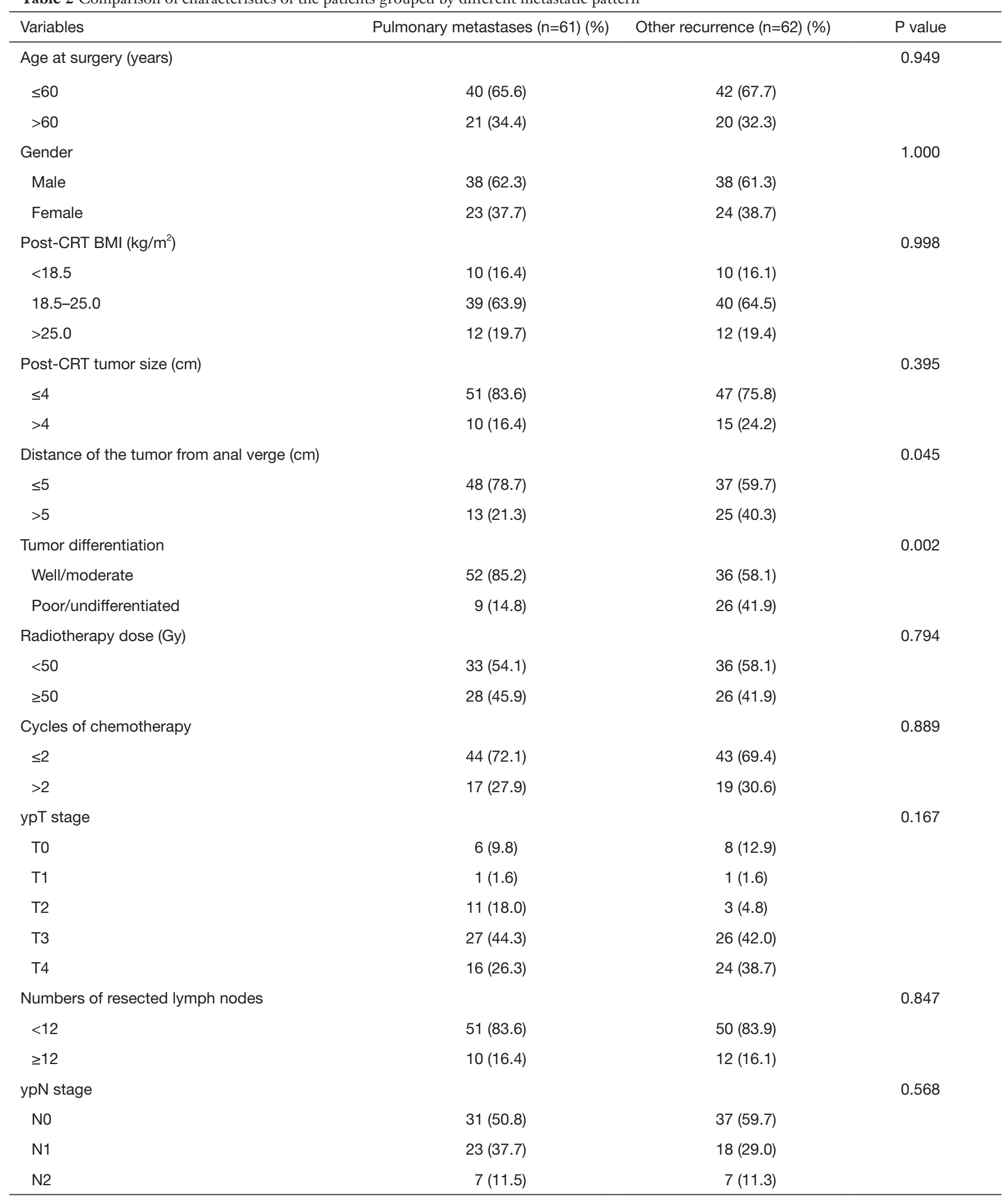

Table 2 (Continued) 
Table 2 (Continued)

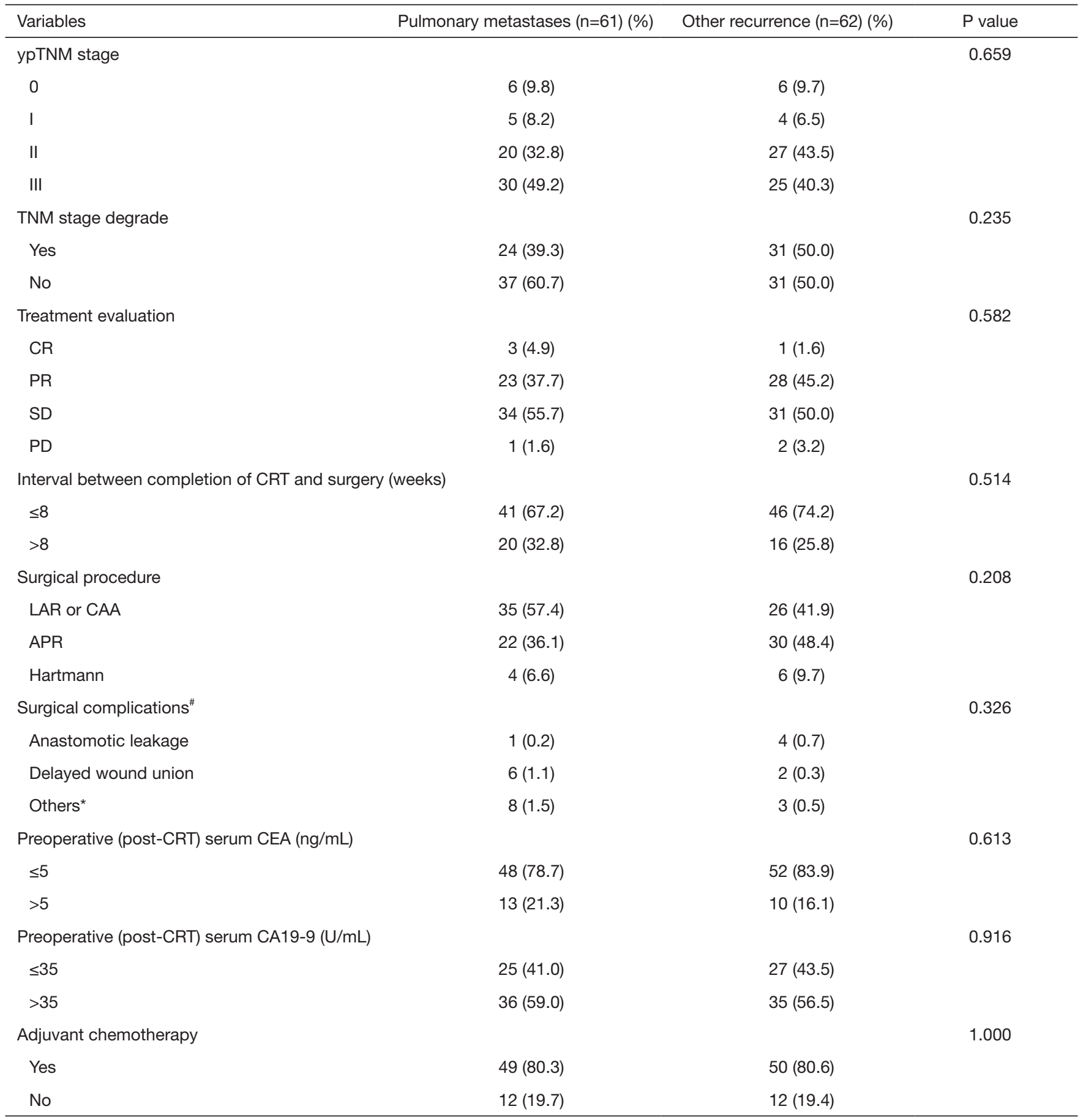

Notes: other recurrence group includes patients with other extrathoracic metastases or local recurrence as the first disease progression after surgery. \#, there were totally 15 patients $(2.8 \%)$ in pulmonary metastases group and 9 patients $(1.5 \%)$ in other recurrence group developing surgical complications; *, other surgical complications include anastomotic bleeding, obstruction, perforation, sexual dysfunction, defecation disorder, Uropoiesis dysfunction. CRT, chemoradiotherapy; BMI, body mass index; CR, complete response; PR, partial response; SD, stable disease; PD, progressive disease; LAR, low anterior resection; CAA, coloanal anastomosis; APR, abdominoperineal resection; CEA, carcinoembryonic antigen; CA19-9, carbohydrate antigen 19-9. 
Table 3 Uni- and multivariate analysis of risk factors for pulmonary metastases [3-year pulmonary metastasis rate (\%)] after neoadjuvant chemoradiotherapy and $\mathrm{R} 0$ resection of locally advanced rectal cancer

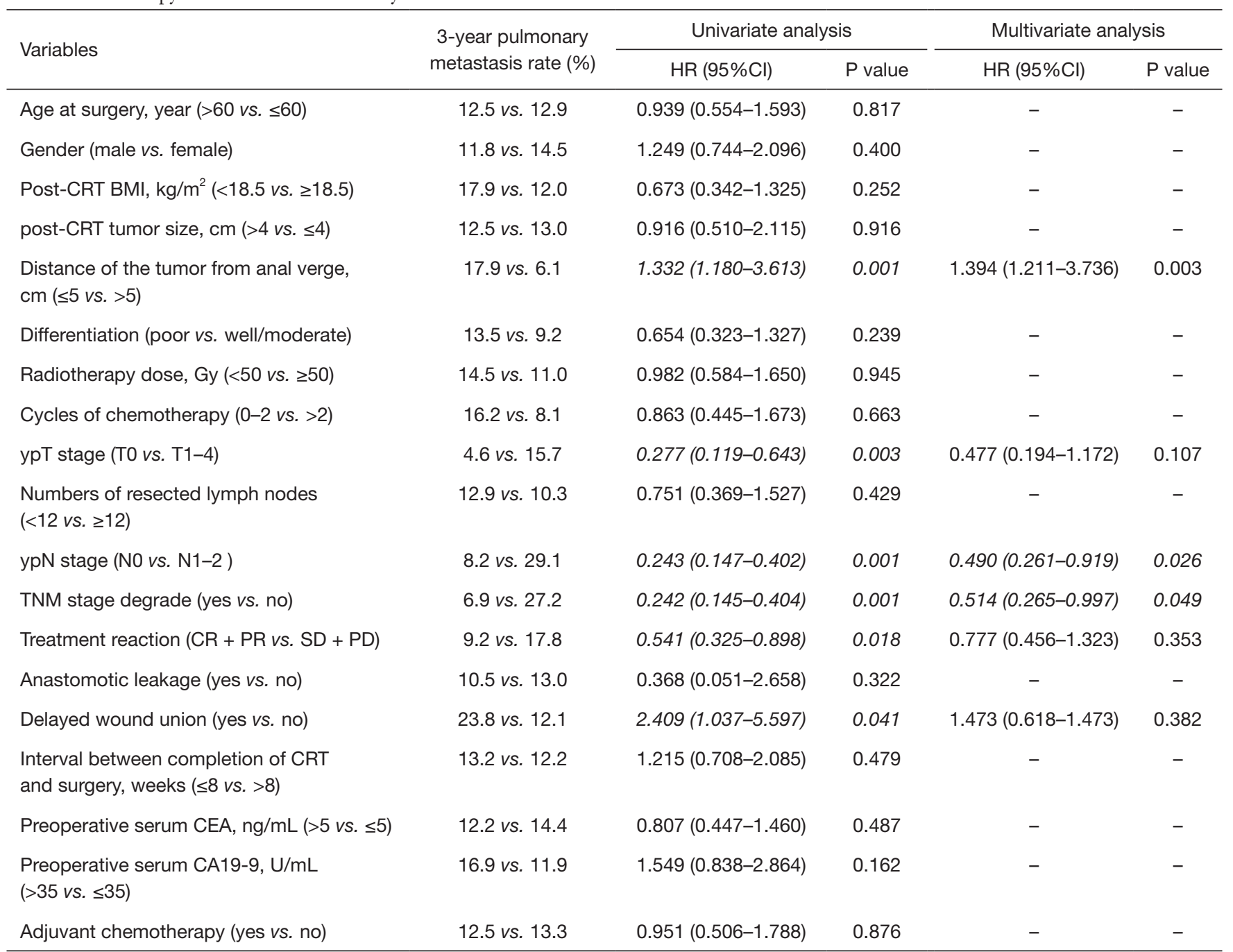

Notes: 62 patients with other extrathoracic metastases or local recurrence as the first disease progression after surgery (other recurrence group) were excluded. CRT, chemoradiotherapy; BMI, body mass index; CR, complete response; PR, partly response; SD, stable disease; $\mathrm{PD}$, progressive disease CEA, carcinoembryonic antigen; CA19-9, carbohydrate antigen 19-9; HR, hazard ratio; Cl, confidence interval.

free group (39.3\% vs. $76.5 \% ; \mathrm{P}<0.001 ; 42.6 \%$ vs. $61.5 \%$; $\mathrm{P}=0.037 ; 9.8 \%$ vs. $28.5 \%$; $\mathrm{P}<0.001$ ), while the rates were similar between the pulmonary metastases group and other recurrence group. There were more patients in the pulmonary metastases group with a preoperative postCRT serum CEA level $>5 \mathrm{ng} / \mathrm{mL}$ than were found in the disease-free group $(21.3 \%$ vs. $5.0 \%$; $\mathrm{P}<0.001)$. Patients in the pulmonary metastases group had better tumor differentiation than was found in those in other recurrence group $(85.2 \%$ vs. $58.1 \% ; \mathrm{P}=0.002)$. There were no significant differences in age, sex, post-CRT BMI, tumor size, the radiotherapy dose, the number of resected lymph nodes, the interval between CRT and surgery, the surgery procedure type, surgical complications, preoperative postCRT serum CA19-9 levels, or the adjuvant chemotherapy rate.

\section{Prognostic analysis of clinical factors}

As shown in Table 3, univariate analysis revealed that the distance of the tumor from the anal verge, ypT stage, ypN stage, TNM downstaging, response, and wound delayed 
union were significant risk factors for the 3-year pulmonary metastasis rate. In the multivariate analysis, a distance of the tumor from anal verge $\leq 5 \mathrm{~cm}$ (HR, 1.394; 95\% CI, 1.2113.736; $\mathrm{P}=0.003$ ) was identified as an independent negative predictor of the 3-year pulmonary metastasis rate, and the N0 stage (HR, 0.490; 95\% CI, 0.261-0.919; $\mathrm{P}=0.026$ ) and TNM downstaging (HR, 0.514; 95\% CI, 0.265-0.997; $\mathrm{P}=0.049)$ were identified as independent positive predictors of the 3-year pulmonary metastasis rate.

\section{Discussion}

In this retrospective study, we investigated risk factors for the development of rectal cancer pulmonary metastases after preoperative CRT and TME. We found that patients with low rectal cancer, lymph node metastases and a poor response to preoperative CRT were more likely to develop pulmonary metastasis.

The benefit of neoadjuvant CRT in decreasing local recurrence and distant metastases has been well-established and was reported by Sauer et al. (11). In the present study, significantly higher proportions of downstaging and $\mathrm{pCR}$ were found in the disease-free group than in the pulmonary metastases group. Moreover, downstaging was identified as an independent positive predictor of the 3-year pulmonary metastasis rate in the multivariate analysis, showing that the response to neoadjuvant CRT is associated with a reduced risk of developing pulmonary metastases after surgery. As reported by $\mathrm{Lu}$ et al. (22), the estimated 5 -year OS was significantly better in responders than in non-responders (94\% vs. $68 \%, \mathrm{P}=0.001$ ), and there was also a significant difference in the 3-year DFS between the two groups ( $93 \%$ vs. $68 \%, \mathrm{P}=0.000)$. Most studies suggest that there are improved outcomes with tumor regression and complete remission to preoperative therapy (23-27). A randomized trial that compared patients with pCR to patients with non-pCR showed that the 5-year disease-specific survival increased from $78.0 \%$ to $95.8 \%$ (28). Chemoradiotherapy leads to microvascular damage and fibrosis of the tumor, blocking the pathway leading to pulmonary metastasis. One explanation for the good prognosis observed after pCR is that it is indicative of a prognostically favorable biological tumor profile with a lower propensity for local recurrence and distant metastases and better survival than is achieved in patients with a lesser response (29).

Patients with primary tumors located in the low rectum or lymph node metastases had a higher risk of developing pulmonary metastases in our study. A study of 10,398 CRC survivors found that the highest risk factors associated with recurrent CRC isolated pulmonary metastases were a low location of a rectal tumor and positive lymph nodes (30). Ding et al. showed that the tendency to develop pulmonary metastases was significantly higher for a low location of rectal tumors than for a higher location of rectal tumors (31). The reason that low rectal tumors are related to a higher pulmonary metastases rate is probably associated with the anatomical structure. The distal rectum drains into the inferior rectal vein, which drains directly into the inferior vena cava so that blood quickly reaches the lungs without passing the portal venous system. Several studies have reported the lymph node ratio (ratio of metastatic lymph nodes to resected lymph nodes) is a predictor of distant metastases after resection of rectal cancer with or without preoperative chemoradiotherapy (32-34). Interestingly, mediastinal lymph node metastases at the time of pulmonary metastasectomy have been shown to have an adverse effect on prognosis (35). Tumor cells may pass directly into lung tissue via the vena cava, and the lymphatic system is another potential route. Local tumor progression may directly affect the incidence of pulmonary metastases in rectal cancer. Therefore, preoperative CRT can effectively reduce the risk of pulmonary metastasis but does not change the pathways associated with and risk factors of pulmonary metastasis.

According to the most recent NCCN guidelines on rectal cancer, the standard follow-up protocol for patients with LARC is chest/abdominal/pelvic CT every 6-12 months for a total of 5 years. The current study found that the incidence of pulmonary metastases rose the fastest during the period from 12-18 months after surgery. Fortyfive patients $(73.8 \%)$ developed pulmonary metastases in the first 24 months. Onaitis and coworkers described a series of 378 patients with colorectal tumor pulmonary metastases whose median disease-free interval was 24 months from the time of the primary operation and demonstrated that a disease-free interval of less than 12 months was an independent negative prognostic factor (36). A retrospective analysis of 153 patients showed that 40 patients $(28.4 \%)$ developed pulmonary metastases in the first 12 months, and 112 patients (79.4\%) developed pulmonary metastases in the first 36 months (37). A review concluded that the median disease-free interval of patients without preoperative CRT ranged from 19 to 38 months and that earlier metastasis might indicate a more aggressive disease biology and tendency to spread (38). In our study, LARC patients with pulmonary metastases after preoperative CRT presented some characteristics, such as a shorter disease-free interval, 
a high proportion $(19.7 \%)$ of concurrent extrathoracic metastases or local recurrence and a high proportion (59.0\%) of bilateral multiple pulmonary metastases, that indicated a prognostically unfavorable biological tumor profile. We suggest that follow-up protocols for LARC patients with a poor response to preoperative CRT should include 6-monthly chest CT examinations for at least 1-2 years after surgery, which is particularly important for patients with low tumors.

Several limitations should be acknowledged in the present study. First, this retrospective study included an uncontrolled methodology and a limited number of patients, especially for patients with pulmonary metastases, recruited from a single cohort. The findings need to be validated in future prospective clinical studies. Additionally, tumor molecular markers, such as microsatellite status, CpG island methylator phenotype (CIMP) status, BRAF mutations, and KRAS mutations, as well as tumor immune infiltration, have been linked to different recurrence risks and survival outcomes in patients with rectal cancer. Thus, it is necessary to include pathological, immunological and molecular prognostic markers for risk stratification in further studies.

In conclusion, pulmonary metastases are more likely to develop in patients with low rectal cancer, lymph node metastases and/or poor responses to preoperative CRT. Detailed preoperative comprehensive measurements might help stratify high-risk patients to identify those who warrant a more intensive follow-up within 2 years.

\section{Acknowledgments}

We deeply appreciate the help we received from all colleagues of the Department of Colorectal Surgery at Sun Yat-sen University Cancer Center who were involved in performing the treatments used in the current study. The authenticity of this article has been validated by uploading the key raw data onto the Research Data Deposit public platform (http://www.researchdata.org.cn), with the Approval Number as RDDA2019001179.

Funding: This work was funded by grants from the National Natural Science Foundation of China (No. 81772595), Science and Technology Planning Project of Guangdong Province (grant No. 2013B090800047), Sun Yat-sen University Clinical Research 5010 Program (No. 2015024 and No. 2013013), and Guangzhou Science and Technology Plan Projects (Health Medical Collaborative Innovation Program of Guangzhou) (grant No. 201803040019).

\section{Footnote}

Conflicts of Interest: The authors have no conflicts of interest to declare.

Ethical Statement: The authors are accountable for all aspects of the work in ensuring that questions related to the accuracy or integrity of any part of the work are appropriately investigated and resolved. The present study was performed according to the ethical standards of the World Medical Association Declaration of Helsinki and approved by the Institutional Review Board and Independent Ethics Committees of Sun Yat-sen University Cancer Center. The informed consent requirement was waived based on the nature of this retrospective study, in which patient data were kept confidential.

\section{References}

1. Siegel RL, Miller KD, Jemal A. Cancer Statistics, 2017. CA Cancer J Clin 2017;67:7-30.

2. Chen W, Zheng R, Baade PD, et al. Cancer statistics in China, 2015. CA Cancer J Clin 2016;66:115-32.

3. Mitry E, Guiu B, Cosconea S, et al. Epidemiology, management and prognosis of colorectal cancer with lung metastases: a 30-year population-based study. Gut 2010;59:1383-8.

4. Tampellini M, Ottone A, Bellini E, et al. The role of lung metastasis resection in improving outcome of colorectal cancer patients: results from a large retrospective study. Oncologist 2012;17:1430-8.

5. Wang Z, Wang X, Yuan J, et al. Survival Benefit of Palliative Local Treatments and Efficacy of Different Pharmacotherapies in Colorectal Cancer With Lung Metastasis: Results From a Large Retrospective Study. Clin Colorectal Cancer 2018;17:e233-55.

6. Siegel R, Desantis C, Jemal A. Colorectal cancer statistics, 2014. CA Cancer J Clin 2014;64:104-17.

7. Siegel RL, Miller KD, Fedewa SA, et al. Colorectal cancer statistics, 2017. CA Cancer J Clin 2017;67:177-93.

8. Zhu J, Tan Z, Hollis-Hansen K, et al. Epidemiological Trends in Colorectal Cancer in China: An Ecological Study. Dig Dis Sci 2017;62:235-43.

9. Zhang Y, Chen Z, Li J. The current status of treatment for colorectal cancer in China: A systematic review. Medicine (Baltimore) 2017;96:e8242.

10. Nordholm-Carstensen A, Krarup PM, Jorgensen LN, et al. Occurrence and survival of synchronous pulmonary 
metastases in colorectal cancer: a nationwide cohort study. Eur J Cancer 2014;50:447-56.

11. Sauer R, Becker H, Hohenberger W, et al. Preoperative versus postoperative chemoradiotherapy for rectal cancer. N Engl J Med 2004;351:1731-40.

12. Bosset JF, Collette L, Calais G, et al. Chemotherapy with preoperative radiotherapy in rectal cancer. $\mathrm{N}$ Engl J Med 2006;355:1114-23.

13. Sebag-Montefiore D, Stephens RJ, Steele R, et al. Preoperative radiotherapy versus selective postoperative chemoradiotherapy in patients with rectal cancer (MRC CR07 and NCIC-CTG C016): a multicentre, randomised trial. Lancet 2009;373:811-20.

14. Pucciarelli S, Gagliardi G, Maretto I, et al. Long-term oncologic results and complications after preoperative chemoradiotherapy for rectal cancer: a single-institution experience after a median follow-up of 95 months. Ann Surg Oncol 2009;16:893-9.

15. Kusters M, Marijnen CA, van de Velde CJ, et al. Patterns of local recurrence in rectal cancer; a study of the Dutch TME trial. Eur J Surg Oncol 2010;36:470-6.

16. Gérard JP, Conroy T, Bonnetain F, et al. Preoperative radiotherapy with or without concurrent fluorouracil and leucovorin in T3-4 rectal cancers: results of FFCD 9203. J Clin Oncol 2006;24:4620-5.

17. Smith KD, Tan D, Das $\mathrm{P}$, et al. Clinical significance of acellular mucin in rectal adenocarcinoma patients with a pathologic complete response to preoperative chemoradiation. Ann Surg 2010;251:261-4.

18. de Campos-Lobato LF, Stocchi L, da Luz Moreira A, et al. Pathologic complete response after neoadjuvant treatment for rectal cancer decreases distant recurrence and could eradicate local recurrence. Ann Surg Oncol 2011;18:1590-8.

19. Garcia-Aguilar J, Smith DD, Avila K, et al. Optimal timing of surgery after chemoradiation for advanced rectal cancer: preliminary results of a multicenter, nonrandomized phase II prospective trial. Ann Surg 2011;254:97-102 .

20. Lin J, Peng J, Qdaisat A, et al. Severe weight loss during preoperative chemoradiotherapy compromises survival outcome for patients with locally advanced rectal cancer. J Cancer Res Clin Oncol 2016;142:2551-60.

21. Prasanna T, Karapetis CS, Roder D, et al. The survival outcome of patients with metastatic colorectal cancer based on the site of metastases and the impact of molecular markers and site of primary cancer on metastatic pattern. Acta Oncol 2018;57:1438-44.

22. Lu JY, Xiao Y, Qiu HZ, et al. Clinical outcome of neoadjuvant chemoradiation therapy with oxaliplatin and capecitabine or 5-fluorouracil for locally advanced rectal cancer. J Surg Oncol 2013;108:213-9.

23. Rödel C, Martus P, Papadoupolos T, et al. Prognostic significance of tumor regression after preoperative chemoradiotherapy for rectal cancer. J Clin Oncol 2005;23:8688-96.

24. Lim SB, Yu CS, Hong YS, et al. Failure patterns correlate with the tumor response after preoperative chemoradiotherapy for locally advanced rectal cancer. J Surg Oncol 2012;106:667-73.

25. Pucciarelli S, Toppan P, Friso ML, et al. Complete pathologic response following preoperative chemoradiation therapy for middle to lower rectal cancer is not a prognostic factor for a better outcome. Dis Colon Rectum 2004;47:1798-807.

26. Chan AK, Wong A, Jenken D, et al. Posttreatment TNM staging is a prognostic indicator of survival and recurrence in tethered or fixed rectal carcinoma after preoperative chemotherapy and radiotherapy. Int $\mathrm{J}$ Radiat Oncol Biol Phys 2005;61:665-77.

27. Capirci C, Valentini V, Cionini L, et al. Prognostic value of pathologic complete response after neoadjuvant therapy in locally advanced rectal cancer: long-term analysis of 566 ypCR patients. Int J Radiat Oncol Biol Phys 2008;72:99-107.

28. Belluco C, De Paoli A, Canzonieri V, et al. Long-term outcome of patients with complete pathologic response after neoadjuvant chemoradiation for cT3 rectal cancer: implications for local excision surgical strategies. Ann Surg Oncol 2011;18:3686-93.

29. Maas M, Nelemans PJ, Valentini V, et al. Long-term outcome in patients with a pathological complete response after chemoradiation for rectal cancer: a pooled analysis of individual patient data. Lancet Oncol 2010;11:835-44.

30. Augestad KM, Bakaki PM, Rose J, et al. Metastatic spread pattern after curative colorectal cancer surgery. A retrospective, longitudinal analysis. Cancer Epidemiol 2015;39:734-44.

31. Ding P, Liska D, Tang P, et al. Pulmonary recurrence predominates after combined modality therapy for rectal cancer: an original retrospective study. Ann Surg 2012;256:111-6.

32. Rullier A, Laurent C, Capdepont M, et al. Lymph nodes after preoperative chemoradiotherapy for rectal carcinoma: number, status, and impact on survival. Am J Surg Pathol 2008;32:45-50.

33. Peschaud F, Benoist S, Julie C, et al. The ratio of 
metastatic to examined lymph nodes is a powerful independent prognostic factor in rectal cancer. Ann Surg 2008;248:1067-73.

34. Watanabe K, Saito N, Sugito M, et al. Predictive factors for pulmonary metastases after curative resection of rectal cancer without preoperative chemoradiotherapy. Dis Colon Rectum 2011;54:989-98.

35. Iida T, Nomori H, Shiba M, et al. Prognostic factors after pulmonary metastasectomy for colorectal cancer and rationale for determining surgical indications: a retrospective analysis. Ann Surg 2013;257:1059-64.

Cite this article as: Li W, Peng J, Li C, Yuan L, Fan W, Pan Z, Wu X, Lin J. Prognosis and risk factors for the development of pulmonary metastases after preoperative chemoradiotherapy and radical resection in patients with locally advanced rectal cancer. Ann Transl Med 2020;8(4):117. doi: 10.21037/ atm.2019.12.108
36. Onaitis MW, Petersen RP, Haney JC, et al. Prognostic factors for recurrence after pulmonary resection of colorectal cancer metastases. Ann Thorac Surg 2009;87:1684-8.

37. Yedibela S, Klein P, Feuchter K, et al. Surgical management of pulmonary metastases from colorectal cancer in 153 patients. Ann Surg Oncol 2006;13:1538-44.

38. Gonzalez M, Ris HB, Krueger T, et al. Colorectal cancer and thoracic surgeons: close encounters of the third kind. Expert Rev Anticancer Ther 2012;12:495-503. 